\title{
Optical Technologies for Terabit/s-throughput Feeder Link
}

\author{
Rudolf Saathof, Remco den Breeje, Wimar Klop, Stefan Kuiper, Niek Doelman, Federico Pettazzi, Amir Vosteen, \\ Niel Truyens, Will Crowcombe, Jet Human, Ivan Ferrario \\ Opto-Mechatronics and Space Systems \\ TNO Technical Sciences \\ Delft, the Netherlands \\ Rudolf.Saathof@TNO.nl
}

Ramon Mata Calvo, Juraj Poliak, Ricardo Barrios, Dirk Giggenbach, Christian Fuchs, Sandro Scalise Institute of Communications and Navigation

Satellite Networks Department

German Aerospace Center (DLR)

Oberpfaffenhofen-Wessling, Germany

\begin{abstract}
To overcome data rate limitations of RF communication links with satellites, TNO and DLR envision optical free-space communication feeder links for next generation high throughput satellites. This paper provides a feasibility assessment of such links and the technology needed. The main results of the link budget and the turbulence modeling of terabit/s optical links are presented. Based on these parameters, requirements and status of the link-subsystems are discussed, and a roadmap is presented, aimed at achieving terabit per second optical feeder links.
\end{abstract}

Keywords-Optical sattellite communication, Adaptive optics

\section{INTRODUCTION}

Satellite communications based on radio frequency (RF) will soon reach its ceiling with respect to achievable data rates. In particular the increasing demand of data broadcasting and the future Internet-of-Things will require substantial data rates. The $\mathrm{RF}$ spectrum is a scarce resource and obtaining a license for its usage is becoming more and more cumbersome and expensive. To increase the system capacity, optical free-space communications has been proposed as alternative for the feeder link, i.e. the link between the ground station and the geostationary telecommunication satellite as shown in Figure 1. The use of optical wavelengths is not only an eligible, but also a feasible step [1]-[6].

Although feasibility of optical links have been demonstrated [7], [8], operational ground-to-geostationary communications systems are still future perspective. Several papers describe ground and space segments options, which include both feeder and user links [9]-[12]. The first relay communications system based on optical inter-satellite links, the European Data Relay System (EDRS) [13], is operational since November 2016 [14], providing the Space Data Highway service. Optical communications links between ground and the GEO satellite Alphasat have been performed regularly from the Transportable Adaptive-Optics Ground Station (TAOGS) by Tesat Spacecom [15]. These communication links demonstrate the feasibility of high data rates through the atmosphere. DLR demonstrated in October 2016 the feasibility of dense-division-multiplexing (DWDM), a fiber based technology, in a GEO-equivalent turbulent channel, setting the world record of 1,72 Terabit/s in free-space optical communications [16]. A practical technical implementation with larger series of ground terminals still is missing, to provide a worldwide coverage of data provision. Such a system should be tailored to be cost efficient, maintenance limited and versatile.

The main channel impairments for optical communications are cloud coverage and atmospheric turbulence. Cloud coverage can be solved with a network of multiple optical ground stations to create site-diversity [9]-[11], [17]. Atmospheric turbulence induces strong signal fluctuations that can strongly degrade communication performance and has large impact on the link budget. To improve the communications performance and optimize the required optical transmit power, an adaptive optics (AO) system is proposed to mitigate the turbulent effects of the

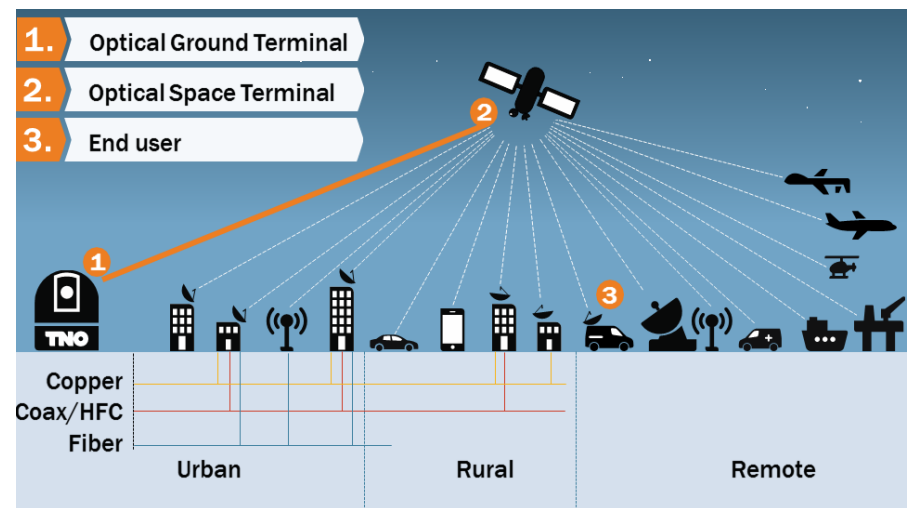

Figure 1 Optical feeder link system architecture. Optical ground stat stations feed the satellite with data. The satellite connects the users via RF links. 
optical channel [18]. The main components of an AO system are a wavefront sensor (WFS) [19], a deformable mirror (DM) and a control system (CS) [20].

This paper presents the approach for a feasible Terabit/s optical feeder link (OFL) system architecture with the goal of a near-future realization for high data rate satellite communications.

In Section II the OFL system architecture, together with the optical channel and the turbulence compensation system are presented. In Section III.A first the turbulence modeling results are presented. Using the network constellation and channel architecture, a link budget is devised in Section III.B using the turbulence modeling, the link budget subsystem requirements are derived in Section IV and compared with the current status. To give a future outlook, a roadmap is added in Section V to achieve a Terabit/s OFL system.

\section{OFL SYSTEM ARCHITECTURE}

In order to share vision and provide ground to estimate feasibility, in this section the OFL system architecture is presented.

\section{A. OFL system architecture}

The main challenge for telecom satellites will be to provide the capacity sufficient for the growing Internet-of-Things and a global broadband access to Internet. Figure 1 shows a satellite communications system with an OFL. The optical ground terminals (OGTs) feed the satellite through the optical feeder link, which is especially challenging due to the aggregate throughput that is in the order of Terabit/s. The satellite connects to the users through many RF user links. Both feeder and user links are bidirectional.

In order to guarantee the required telecommunications availability, multiple OGTs are foreseen to avoid cloud coverage. Several studies investigated the number OGTs which are needed to compensate for the cloud blockage [9]-[11]. It can be concluded that with 12 OGS sites in European countries more than $99.9 \%$ system availability ("at least one gateway location is cloud-free") can be warranted [4]. In this case, only one active OGT is assumed, which provides the necessary throughput to the satellite. With a wider ground network, the use of multiple active OGT links with one satellite is considered. Multiple payload optical heads operated in parallel with individual links could distribute the data throughput load, at the cost of expanding the ground network to keep the same availability on system level and a higher complexity on board the satellite.

The OFL communications architecture will support the DVB-S2X broadcasting standard, to allow the satellite to implement the RF user links. The DVB standards describe the modulation and coding schemes for broadband satellite communication links. Several architectures are possible, depending on the signal processing on board of the satellite. A digital architecture has the advantage of allowing an error correction on the satellite, to protect the feeder link from the channel impairments. A regenerative architecture may reach better performance because the communications system can be optimized. However, usually a transparent architecture is preferred because allows future upgrades of the communications

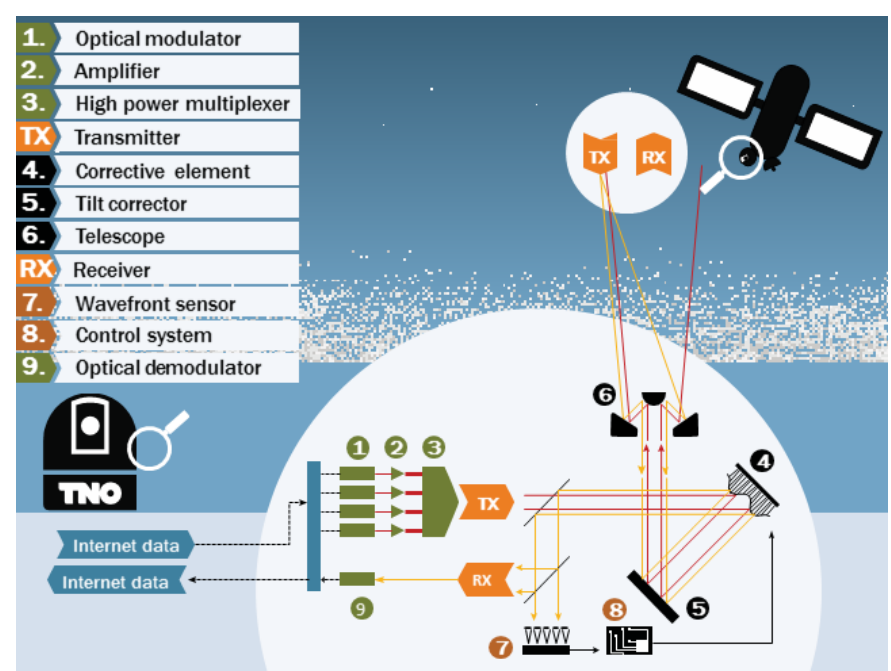

Figure 2 Basic architecture of an Adaptive optics system for optical turbulence mitigation of turbulence effects.

system. An accurate design of the coding and the signal processing distribution between OGT and satellite has to be done to guarantee the maximum communications performance. In the design, the on-board processing capabilities need to be accurately taken into account to minimize the complexity and consumption of the satellite communications payload.

The main elements of the communications system are represented in Figure 3. The interface with the optical network to accommodate the data into the optical channels (including any coding or data-preprocessing for the feeder link and the DVBS2X data format conversion when required), the modulation of the optical channels (coherent/non-coherent), multiplexing and amplification for the gateway side. In the satellite segment, the main elements in the communications chain are the preamplification, de-multiplexing, the detection (with a hybrid when coherent modulations formats are used and eventually an phase-lock loop for homodyne reception), the signal processing and interface towards the frequency conversion (including processing for the data reception and any decoding scheme for digital architectures) and finally the conversion to the Ka-Band for the user-link.

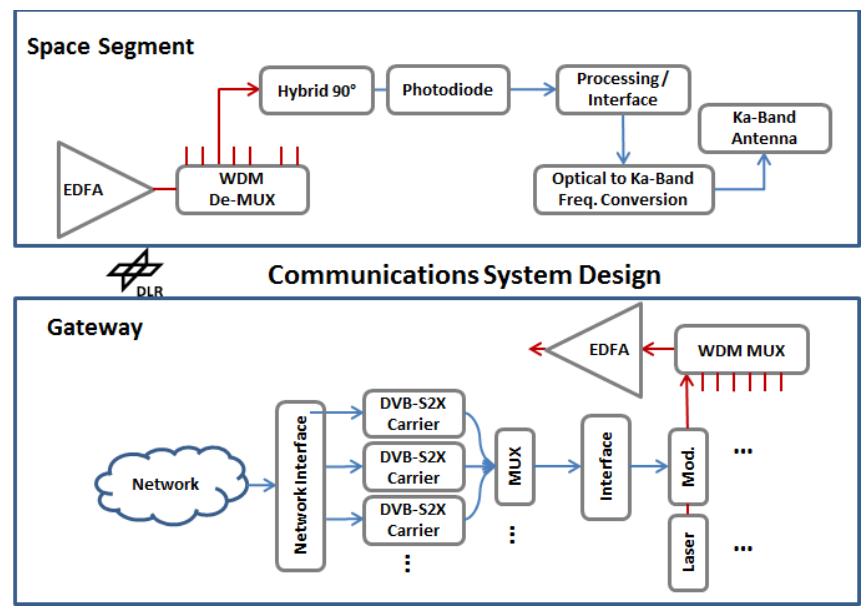

Figure 3. Main elements of the communications system 


\section{B. Optical system architecture}

Figure 2 presents the baseline conceptual architecture for the OGT, based on a monostatic concept, in which both transmitter and receiver channels share the same output optical aperture and part of the optical path.

On one end, the downlink beam is collected by the telescope and relayed via a tip-tilt corrector and a deformable mirror to a section which separates the receiving beam from the transmitted beam. The receiving beam is routed to the sensing part, which is composed by of both a tip-tilt sensor and a wavefront sensor. On the other end, light generated by the Tx is routed via a pointahead mirror to the tip-tilt corrector and the deformable mirror and relayed to the telescope, to be transmitted to the satellite terminal. During operation in closed-loop, the wavefront measurement of the downlink is used for the pre-correction of the wavefront of the uplink beam, to partially compensate the losses due to propagation through turbulent atmosphere.

In the current baseline, uplink and downlink beams differ in both polarization and wavelength. More specifically, they propagate through the atmosphere with opposite circular polarization, so that separation between the two is performed by means of a quarter-wave plate and a polarizing beam splitter. Furthermore, a guard band of at least $10 \mathrm{~nm}$ will be introduced between the Tx and Rx channels, which provides additional improvement of separating the uplink and downlink channels.

The telescope subsystem is one of the critical parts of the OGT. It should be able to project relatively high power laser beams towards the OST, receive the downlink beam, and provide at the same time the beacon laser and coarse pointing of the communications and beacon beams. In the current baseline, an afocal off-axis system in a Coudé configuration is anticipated. This configuration is judged more adequate to handle the expected high power beam from the Tx channel and will guarantee at the same time a reduced backscattering towards the receiver path.

The AO system will be based on standard folded $4 \mathrm{f}$ relays, in which the system entrance pupil is re-imaged multiple times across the optical path onto planes coincident with actuators (i.e. deformable mirror, fine-steering mirror and point-ahead mirror) and sensing devices (Shack Hartman sensor and tip-tilt sensor). Both deformable mirror and fine-steering mirror will be placed in the common path, shared between the Tx and Rx beam. In such a way, they will perform correction of both downlink and uplink beam, thus improving both receiver fiber coupling efficiency [21] and transmitter propagation through the atmosphere [22], [23]. Applying the pre-correction not only reduces the scintillation, but also the beam wander. This would allow for a release of the beam divergence constraint in the link budget. This way, the pre-distortion AO system on the ground station drastically reduces the required transmitted power per channel.

The photonics subsystem is responsible for the generation of the modulated high power laser beam which will be transmitted across the atmosphere, as well as for the conversion of the downlink beam into an RF signal. In the current baseline, both uplink and downlink beams will belong to the telecom $\mathrm{C}$ (and potentially L) band, which will allow the use of components
Table 1 Turbulence and channel parameters

\begin{tabular}{|c|c|c|c|}
\hline Beam parameter & best & median & worst \\
\hline Isoplanatic angle up [ $\mu \mathrm{rad}]$ & 59.1 & 16.8 & 8.27 \\
\hline Beam Wander up RMS [ $\mu \mathrm{rad}]$ & 1.30 & 3.70 & 6.93 \\
\hline Beam spot size up [m] & 137 & 223 & 402 \\
\hline $\begin{array}{l}\text { Fried Coherence length down } \\
{[\mathrm{cm}]}\end{array}$ & 110 & 31.2 & 14.7 \\
\hline $\begin{array}{l}\text { Angle-of-Arrival downlink RMS } \\
\text { [ } \mu \mathrm{rad}]\end{array}$ & 0.732 & 2.09 & 3.91 \\
\hline $\begin{array}{l}\text { Wavefront Phase error down } \\
\text { RMS [rad] }\end{array}$ & 0.344 & 0.982 & 1.84 \\
\hline Greenwood frequency [Hz] & 7.40 & 18.7 & 30.6 \\
\hline $\begin{array}{l}\text { Aperture Averaged scintillation } \\
\text { down }\end{array}$ & 0.001 & 0.011 & 0.037 \\
\hline $\begin{array}{l}\text { Longitudinal scintillation up } \\
\text { (tracked beam) }\end{array}$ & 0.015 & 0.094 & 0.219 \\
\hline
\end{tabular}

developed for the telecom market. In this respect, it should be noted that both the Tx and Rx sections of the photonic subsystem will rely on the Erbium-doped amplifier technology, a versatile platform that can be used to both to boost the power of the Tx beam, to the power levels given by the link budget, as well as to amplify the low-level downlink beam with low noise factors.

\section{MODELING RESULTS}

With this architecture in mind, modeling of the optical turbulence and the optical power has been performed. A link budget is devised, which represents the optical power budget over the link.

\section{A. Turbulence modeling results}

TNO has developed models and correction strategies to estimate the turbulence-induced wavefront errors [24] and to optimize the performance of the AO pre-correction. To properly describe the entire optical system, both the uplink and the downlink have been modeled.

Because the theory of turbulence modeling were studied extensively in many publications [25]-[28], and many other sources have applied these in their publications [22], [29]-[31], it is sufficient to describe the boundary conditions and the modeling approach that is taken. In addition, the main results will be given.

The complete system of ground terminals and space terminals is considered. It is desired to estimate the expected turbulence parameters of all combinations of ground terminals and space terminals. To describe the turbulences, beam distortion is studied for both uplink and downlink, based on the following inputs:

- A set of 25 Southern-European potential OGT locations ranging from Porto to Nicosia and including Tenerife.

- Three GEO-satellite slots (at longitudes $8^{\circ} \mathrm{W}, 9^{\circ} \mathrm{E}$ and $\left.31^{\circ} \mathrm{E}\right)$

- Three types of turbulence conditions; using the HufnagelValley model with the following parameters: 
a) Weak turbulence: $C_{n}{ }^{2}(0)=1 \mathrm{e}-15 \mathrm{~m}^{-2 / 3}$, pseudo-wind $W=10 \mathrm{~m} / \mathrm{s}$ and $V_{\text {ground }}=8 \mathrm{~m} / \mathrm{s}$.

b) Moderate: $C_{n}^{2}(0)=1.7 \mathrm{e}-14 \mathrm{~m}^{-2 / 3}, \quad W=21 \mathrm{~m} / \mathrm{s}$ and $V_{\text {ground }}=8 \mathrm{~m} / \mathrm{s}$.

c) Moderate with strong wind: $C_{n}{ }^{2}(0)=1.7 \mathrm{e}-14 \mathrm{~m}^{-2 / 3}$, $W=21 \mathrm{~m} / \mathrm{s}$ and $V_{\text {ground }}=12 \mathrm{~m} / \mathrm{s}$.

- The transmit and receive apertures of the OST and the receive aperture of the OGT are assumed $400 \mathrm{~mm}$ in a first step, to satisfy the link budget. The effective OGT transmit beam is optimized to a diameter of $300 \mathrm{~mm}$ without central obscuration, whereas the OST is assumed to have central obscuration.

This geometry of OGS locations and Satellite slots results in a distribution of turbulence parameters which influence the link budget. A selection of the turbulence and channel parameters is presented in Table 1.

\section{B. Link budget}

For communication links, the system parameters and the performance margins are established by means of the link equation [32], which describes the process of transmission of signal through the atmosphere accounting for all the possible gain and loss terms. The optical link budget defines the sum of the transmitted power and all expected gain and loss contributors, and relates it to the receiver's sensitivity threshold taking into account a margin. This section gives the link budget of an OFL for a very high throughput system to achieve Terabit/s throughput, which is the basis of the OGT product design.

The OFL link budget is defined with the following design decisions and assumptions. The assumptions will be reassessed in the program, updating the link budgets accordingly.

- The uplink and downlink beams consist of multiple wavelength-division multiplexed (WDM) channels in the optical C-Band spectrum for both uplink and downlink data streams.

- The optical receiver sensitivity threshold value is $45.3 \mathrm{dBm}$, the modulation scheme is on off keying (OOK), with a bit error rate (BER) of $10^{-3}$, before forward error correction.

- Using turbulence modelling, losses due to pointing errors, beam spreading and scintillation are estimated. The performance improvement with adaptive optics is expected to be in the order of $20 \mathrm{~dB}$ by correcting the first 30 modes.

- Scintillation losses are obtained using a log-normal distribution and an outage probability of 0.001 [33].

The link budgets given in Table 2 indicate that an OGT requires 300 Watt $(55 \mathrm{dBm})$ power over $30 \mathrm{DWDM}$ channels transmit to provide sufficient optical power at the receiver. This significantly impacts the optical design of the OGT.

\section{STATUS LINK-SYSTEMS}

The most straightforward approach to estimate the number of modes for an AO system is to use the Fried parameter. Here
Table 2 Link budget

\begin{tabular}{|c|c|c|}
\hline Name & Uplink & Unit \\
\hline \multicolumn{3}{|l|}{ Total link parameters } \\
\hline Total power including corrections & $>55$ & $\mathrm{dBm}$ \\
\hline \multicolumn{3}{|l|}{ WDM channel parameters } \\
\hline Optical power Tx (average) & 40 & $\mathrm{dBm}$ \\
\hline Tx diameter & 0.3 & $\mathrm{~m}$ \\
\hline Link distance & $39 \cdot 10^{6}$ & $\mathrm{~m}$ \\
\hline Rx diameter & 0.4 & $\mathrm{~m}$ \\
\hline \multicolumn{3}{|l|}{ Channel link budget } \\
\hline Tx-Optics loss & -2.21 & $\mathrm{~dB}$ \\
\hline TX-Gain & 114.63 & $\mathrm{~dB}$ \\
\hline Wavefront error losses & -0.76 & $\mathrm{~dB}$ \\
\hline Free space losses & -290.00 & $\mathrm{~dB}$ \\
\hline Rx-Gain & 118.17 & $\mathrm{~dB}$ \\
\hline Atmospheric attenuation & -0.40 & $\mathrm{~dB}$ \\
\hline Rx-Optics loss & -2.21 & $\mathrm{~dB}$ \\
\hline Cloud margin (cirrus clouds) & -3.00 & $\mathrm{~dB}$ \\
\hline Turbulence losses (AO corrected) & -11.23 & $\mathrm{~dB}$ \\
\hline Tx power (per channel) & 39.73 & $\mathrm{dBm}$ \\
\hline \multicolumn{3}{|l|}{ Receiving link } \\
\hline $\begin{array}{c}\text { Rx-Power } \\
\text { (target including margin) }\end{array}$ & -37.27 & $\mathrm{dBm}$ \\
\hline Target Rx power (threshold) & -45.3 & $\mathrm{dBm}$ \\
\hline Margin & 8 & $\mathrm{~dB}$ \\
\hline Total link loss & -77.00 & $\mathrm{~dB}$ \\
\hline
\end{tabular}

we aim for correction of the first 30 modes and an effective residual wavefront error of $100 \mathrm{~nm}$ (RMS), which corresponds to slightly better than $\lambda / 14$. Note that this differs from the ruleof-thumb approach to have the DM actuator spacing in the order of the Fried coherence length. In addition, the Greenwood frequency gives an indication of the desired closed-loop bandwidth. The AO system presented here targets a bandwidth of above $100 \mathrm{~Hz}$.

\section{A. Ofelia AO breadboard}

The implementation of high data rate transmission between ground and space relies strongly on the use of AO. It will be used to optimize the fiber coupling of the received downlink beam and at the same time to pre-correct the wavefront of the uplink beam.

The performance of the foreseen $\mathrm{AO}$ architecture will be verified in an early stage of the program by means of a dedicated breadboard plan. For this activity, a terrestrial communication test will be realized between two breadboards. One of the two breadboards, the ground terminal breadboard (GTB), will mimic the ground terminal, and will be equipped with an adaptive optics bench. The space terminal breadboard (STB), will be a representative of the space segment, and its design will be based on previously developed breadboards for high data rate communication tests [26]. 

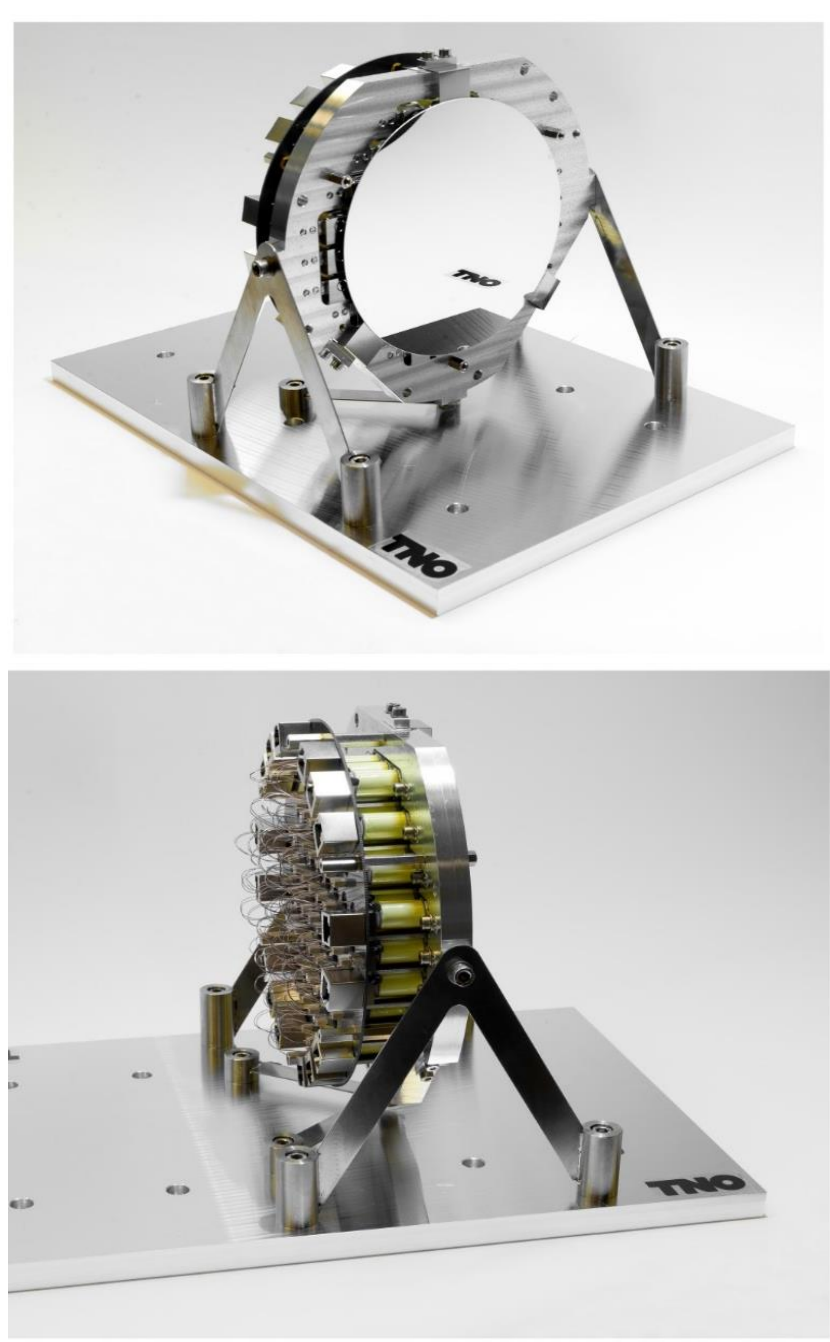

Figure 4 Deformable mirror.

Being focused on the performance of the AO system, the breadboard test will not display any data communication functionality. Instead, it will implement the full AO correction chain for a bi-directional link as described in Section II. Tests will be done to verify its performance in different operational conditions. In particular, the large divergence beacon beam emitted by the STB will be captured by a $90-\mathrm{mm}$ receiving telescope of the GTB and relayed to the diagnostic sensors, which will include a Shack-Hartmann sensor, a focus camera and a tip-tilt sensor. The information retrieved by those sensors will be used to control a deformable mirror and a fine-steering mirror, which will in turn pre-correct the wavefront of a low divergence Tx beam, which represents the uplink channel.

The uplink beam will be received by a relatively small aperture telescope on the STB side $(10 \mathrm{~mm})$ to mimic the relatively large coherent radius of the uplink compared to the GEO satellite aperture. The retrieved signal will be used to evaluate the scintillation suppression achieved via the implementation of the AO pre-correction scheme.

On one side, the selected apertures for the GTB and STB telescopes will make sure, that the received downlink beam will see multiple coherence radii of turbulent atmospheric distortion.

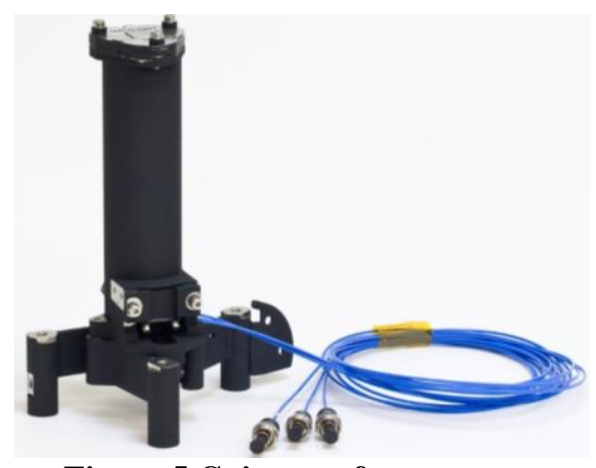

Figure 5 Gaia wavefront sensor.

On the other side, the uplink received beam will have little aperture averaging, so it will provide a realistic measure of the worst-case scintillation effect and its reduction.

One of the key objectives of the breadboard plan is to evaluate the effect of the point-ahead angle on the performance of the AO correction. This is achieved by introducing a pointahead mirror in the GTB, which will be used to apply an offset tilt to the outgoing uplink beam. On the other side of the communication link, the STB will be equipped with a system that allows up to a $1 \mathrm{~m}$ lateral displacement of the receiver telescope. In such a way, a geometry representative of the communication link between ground and space will be simulated, and its impact on the AO correction will be measured.

The breadboard is currently undergoing the critical design phase process, and it is expected to be ready to perform open air tests by the beginning of 2018 .

\section{B. Deformable mirror}

Over the last decade TNO has developed a deformable mirror concept using electro-magnetic actuators based on the principle of variable reluctance. This leads to a DM with a very low non-linearity and hysteresis, low power consumption, and high inherent reliability of the actuators [34]. The increased actuator efficiency gives improvement of the DM performance in terms of dynamical performance, actuation range, and power dissipation.

The realized DM is presented in Figure 4. It has 57 actuators, which is more than sufficient to accommodate for correction of 30 modes. In addition, the resonance frequency of the piston mode is $1.5 \mathrm{kHz}$. Hence, it will be able to fulfill the bandwidth requirement of more than $100 \mathrm{~Hz}$. The inter-actuator stroke is $17 \mu \mathrm{m}$, which is more than sufficient to correct for $1.8 \mathrm{rad}$, which corresponds to a displacement of $2.8 \mu \mathrm{m}$ at the wavelength of $1550 \mathrm{~nm}$.

\section{Wavefront sensor}

TNO developed a Wave Front Sensor (WFS) instrument for the GAIA mission [19]. This Wave Front Sensor is used to monitor the wave front errors of the two main telescopes mounted on the GAIA satellite, which has a 5-degree of freedom (DOF) correction mechanism. The GAIA-WFS operates over a broad wavelength (450 to $900 \mathrm{~nm})$ and under cryogenic conditions (130 to $200 \mathrm{~K}$ operation temperature). The WFS uses an all reflective, a-thermal design and is of the type of Shack- 


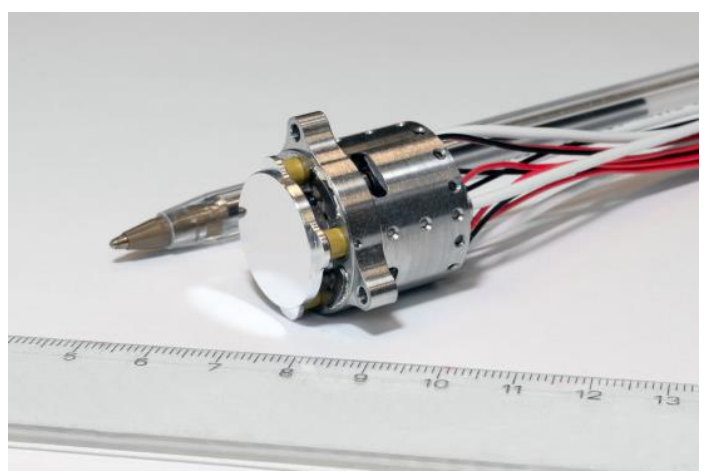

Figure 6 Fast Fast-steering mirror.

Hartmann. The boundary condition for the design is that the focal plane of the WFS is the same plane as the focal plane of the GAIA telescopes. The spot pattern generated after a micro lens array (MLA) by a star is compared to the pattern of one of the three calibration sources that is included in the WFS, allowing in-flight calibration. This WFS has a robust and lightweight opto-mechanical design that is optimized for launch and cryogenic operation (Figure 5).

Whereas the GAIA WFS is working in the visible range under very stringent conditions, for laser satellite communication TNO is developing a dedicated ShackHartmann WFS dedicated for $1550 \mathrm{~nm}$. Hence, it cannot use Silicon-based detector technology. In addition, latency is one the main requirements. Therefore, a high-speed InGaAs detector is selected. To accommodate for more than 30 modes, a lenslet array of 10x10 lenses is selected.

\section{Fast steering mirror}

A Fine-steering mirror (FSM) is developed, dedicated for inter-satellite laser communication, which could also be used for OGTs [35]. This FSM design is targeted at high dynamical performance of $>1 \mathrm{kHz}$ disturbance rejection bandwidth, and an optical angular range of more than $\pm 2^{\circ}$ in two axes. The actuator design is based on the variable reluctance principle, and the tailored for the specific applications. The dynamical performance and integrity under thermal loading have been assessed via FEM modeling showing conformity with the performance and environmental requirements. The resulting FSM design is highly compact and power efficient, making it highly adequate for the evolving field of inter-satellite laser communications and OGTs (see Figure 6).

\section{E. Photonics}

As mentioned in previous section, the photonics subsystem relies on the use of photonics components developed for the terrestrial telecom market and operating in the optical C-band. Among them, the Erbium-doped amplifier (EDFA) is of paramount importance, since it allows reaching the power values required to close the link budget.

The state of the art of EDFA systems used for both transmitters and receiver is compatible with the performance required by the OFL: EDFA booster amplifiers covering the full C-band with output power exceeding $40 \mathrm{dBm}$ are currently available from multiple suppliers, and ultra-low noise receiver

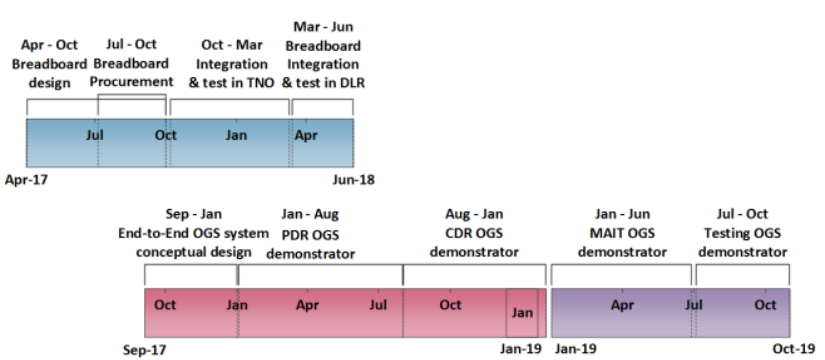

Figure 7 Terabit optical feeder link roadmap.

amplifiers with noise figures approaching the theoretical $3 \mathrm{~dB}$ are also developed.

\section{ROADMAP}

The main goal for the activities described in this paper is the development of an OGT product for use in terabit/s OFLs. The Ofelia breadboard described in Section IV.A will be a significant step to prepare this OGT product. First, it serves for verification of the turbulence models and the link budget. Second, it provides the opportunity to test the adaptive optics and photonics technologies described in Section IV. The timeline of this development plan is presented in Figure 7. With the results of this breadboard experiment, a demonstrator will be designed close to a realistic product. Besides this, OGT product for terabit/s OFLs, also other products are developed, such as terminals for Gbit/s. In this fashion, a complete set of products and technologies are being developed.

To support the technologies development foreseen in the optical communication (OC) domain, TNO is also implementing an OC lab. Such a facility should be used for technology development and to perform proof of principle end to end communication tests for both internal projects, as well for external parties. Furthermore, the intended facility is aimed to allow the development of a quantum optical communication demonstrator compatible with the currently foreseen architecture for high data throughput links. At present three different uses of the facility are foreseen:

- Laboratory testing mode: the facility is used in a reduced mode to allow laboratory testing of the main functionality, and perform a comparison of different subsystem performances;

- End to end reduced distance field testing: the facility is used to perform end to end testing at tens of $\mathrm{km}$ distance with low/medium power laser systems;

- Demonstrator level testing: the facility is used to perform representative/qualification testing for a high throughput Optical Feeder Link.

The design of the facility is currently starting, and a target development period of 2 years is currently foreseen. This facility would not only support the development of technologies, such as mentioned in this paper, but also the transfer of knowledge.

Communications technologies for future very high throughput optical satellite systems are being developed and tested at the DLR premises in Laboratory for terabit throughput satellite system technologies (THRUST-Lab) [36]. Current 
development focuses also on the development of complete optomechanical assembly of demonstrators of possible future satellite missions including the optical, electrical and mechanical subsystems tailored to the specifics of the scenarios. These demonstrators are then tested in relevant conditions emulated by means of several terrestrial testbeds, to which DLR has exclusive access.

\section{CONCLUSIONS}

Optical feeder links is essential to increase data throughput in telecommunication satellites to Terabit/s and beyond. In this paper, an optical feeder link system is presented to support this. The main optical turbulence parameters and a first link budget for a feasible optical communication system are presented. From these models, parameters are derived for the critical technical components. By discussion of these, it is shown that Terabit/s optical feeder links are technically feasible. By the discussion of the roadmap it is clear that a commercially feasible feeder link optical ground terminal may be realized within a few years.

\section{REFERENCES}

[1] Z. Sodnik and M. Sans, "Extending EDRS to Laser Communication from Space to Ground," in Proceedings of International Conference on Space Optical Systems and Applications, 2012, vol. 12, pp. 9-14.

[2] W. Rabinovich et al., "Free-space optical communications research and demonstrations at the US Naval Research Laboratory," Appl. Opt., vol. 54, no. 31, 2015.

[3] N. Perlot et al., "Results of the optical downlink experiment KIODO from OICETS satellite to optical ground station Oberpfaffenhofen (OGS-OP)," Lasers Appl. Sci. Eng., vol. 6457, pp. 645704-645704-8, 2007.

[4] M. Toyoshima et al., "Ground-to-satellite laser communication experiments," IEEE Aerosp. Electron. Syst. Mag., vol. 23, no. 8, pp. 10$18,2008$.

[5] D. Giggenbach, F. Moll, C. Fuchs, and T. de Cola, "Space communications protocols for future optical satellite-downlinks," in 62nd International Astronautical Congress, 2011.

[6] C. Schmidt, M. Brechtelsbauer, and F. Rein, "OSIRIS Payload for DLR's BiROS Satellite," in Conference on Space Optical Systems, 2014.

[7] E. Fischer, T. Berkefeld, and M. Feriencik, "Development, integration and test of a transportable adaptive optical ground station," 2015 IEEE International Conference on Space Optical Systems and Applications., pp. 1-6, 2015.

[8] D. Tröndle et al., "Alphasat-Sentinel-1A optical inter-satellite links: runup for the European data relay satellite system," in Proceedings of SPIE, 2016, vol. 9739, p. 973902.

[9] S. Poulenard, M. Crosnier, and A. Rissons, "Ground segment design for broadband geostationary satellite with optical feeder link," J. Opt. Commun. Netw, vol. 7, no. 4, pp. 325-336, 2015.

[10] A. Gharanjik, K. Liolis, and M. Shankar, "Spatial multiplexing in optical feeder links for high throughput satellites," Signal Inf., 2014.

[11] O. Vidal, B. Roy, S. Dimitrov, R. Barrios, and D. Giggenbach, "Next generation high throuhput satellite system based on optical feeder links." 2015.

[12] D. Giggenbach, E. Lutz, J. Poliak, R. Mata-Calvo, and C. Fuchs, “A Highthroughput Satellite System for Serving whole Europe with Fast Internet Service, Employing Optical Feeder Links," in 9th ITG Symposium, 2015, pp. 1-7.

[13] F. Heine, G. Muhlnikel, H. Zech, S. Philipp-May, and R. Meyer, "The European Data Relay System, high speed laser based data links," in IEEE
Advanced Satellite Multimedia Systems Conference and the 13th Signal Processing for Space Communications Workshop (ASMS/SPSC), 2014, pp. 284-286.

[14] F. Heine, D. Troendle, and C. Rochow, "Progressing towards an operational optical data relay service," in Proceedings of SPIE, 2017, p. 10096.

[15] K. Saucke, F. Heine, and M. Gregory, "The Tesat transportable adaptive optical ground station," Proceedings of SPIE, p. 973906, 2016.

[16] "World record in free-space optical communications," 2016. [Online]. Available: $\quad$ http://www.dlr.de/dlr/en/desktopdefault.aspx/tabid10081/151_read-19914/\#/gallery/24870. [Accessed: 17-May-2017].

[17] C. Fuchs and F. Moll, "Ground station network optimization for space-toground optical communication links," J. Opt. Commun., 2015.

[18] R. Tyson and D. Canning, "Indirect measurement of a laser communications bit-error-rate reduction with low-order adaptive optics," Appl. Opt., vol. 42, no. 21, pp. 4239-4243, 2003.

[19] L. Vosteen and F. Draaisma, "Wavefront sensor for the ESA-GAIA mission," in Proceedings of SPIE, 2009, vol. 7439, pp. 743914-4.

[20] N. Doelman, R. Fraanje, and R. den Breeje, "Real-sky adaptive optics experiments on optimal control of tip-tilt modes," in SPIE Conference on Adaptive Optics Systems II, 2011, vol. 7736.

[21] M. Chen, C. Liu, and H. Xian, "Experimental demonstration of singlemode fiber coupling over relatively strong turbulence with adaptive optics," Appl. Opt., vol. 54, no. 29, pp. 8722-8726, 2015.

[22] N. Leonhard et al., "Real-time adaptive optics testbed to investigate pointahead angle in pre-compensation of Earth-to-GEO optical communication," Opt. Express, vol. 24, no. 12, p. 13157, Jun. 2016.

[23] A. Brady et al., "Experimental validation of phase-only pre-compensation over $494 \mathrm{~m}$ free-space propagation," Opt. Lett., vol. 42, no. 14, pp. 2679 2682, 2017.

[24] R. Fraanje and N. Doelman, "Modeling and prediction of turbulenceinduced wavefront distortions," in Proceedings of SPIE, 2010, vol. 7736, p. 773617.

[25] L. Andrews and R. Phillips, Laser beam propagation through random media. SPIE press, 2005.

[26] J. Hardy, Adaptive Optics for Astronomical Telescopes. Oxford University Press, 1998.

[27] G. A. Tyler, "Bandwidth considerations for tracking through turbulence," J. Opt. Soc. Am. A, vol. 11, no. 1, p. 358, 1994.

[28] R. Tyson, "Adaptive optics and ground-to-space laser communications," Appl. Opt., 1996.

[29] R. Mata Calvo et al., "Transmitter diversity verification on ARTEMIS geostationary satellite," in Proceedings of SPIE, 2014, vol. 8971.

[30] S. Dimitrov, R. Barrios, B. Matuz, and G. Liva, "Digital modulation and coding for satellite optical feeder links with pre-distortion adaptive optics,” Int. J. Satell. Commun. Netw., vol. 34, no. 5, pp. 625-644, 2015.

[31] Z. Sodnik, J. Armengol, and R. Czichy, "Adaptive optics and ESA's optical ground station," in Proceedings of SPIE, 2009, vol. 7464.

[32] M. Katzman, Laser satellite communications. Englewood Cliffs, NJ, Prentice-Hall, Inc., 1987.

[33] D. Giggenbach and H. Henniger, "Fading-loss assessment in atmospheric free-space optical communication links with on-off keying," Opt. Eng., vol. 47, no. 6, p. 69801, 2008.

[34] S. Kuiper and N. Doelman, "Electromagnetic deformable mirror development at TNO," in Proceedings of SPIE, 2016, pp. 991204-7.

[35] S. Kuiper et al., "Hight bandwidth and compact fine steering mirror development for laser communications," in 17the European space mechanisms and tribology symposium, 2017.

[36] J. Surof, J. Poliak, and R. Calvo, "Demonstration of intradyne BPSK optical free-space transmission in representative atmospheric turbulence conditions for geostationary uplink channel," Opt. Lett., vol. 42, no. 11, pp. $2173-2176,2017$. 